\title{
Classification of Composite Power Quality Disturbance Signals Based on HHT and S-Transform
}

\author{
Nanhua Yu, Chuanjian Li, Rui Li \\ Electric Power Research Institute of Guangdong Power Grid \\ Corporation \\ Guangzhou 510080, China \\ E-mail:ynhzy2@163.com
}

Shaoge Yin

Hefei University of Technology

Hefei 230009

E-mail: kejizhiguang.cool@163.com

\author{
Wei Liu \\ Guangdong Power Grid Corporation \\ Guangzhou 510080, China \\ E-mail: liuwei@gd.csg.cn
}

\section{Weiqing Tao}

CSG Smart Science\&Technology Co.,Ltd

Shanghai 201100, China

E-mail: twq@csg.cn

\begin{abstract}
The correct classification of single and composite power quality disturbances is the premise and basis of governance and control of power quality problems. This article extracts the high-frequency and low-frequency characteristics by S-transform of the disturbance signals, combining HHT to extract the instantaneous amplitude of the signal before and after the disturbance. Some characteristic functions is defined as the classification criterions by analyzing the characteristics of the disturbance signals, as to achieve the classification of power quality disturbances. Experimental results show that the fusion of the two methods can accurately detect the categories and characteristics of power quality disturbance signals, the starting and ending time of transient disturbance signals.
\end{abstract}

Keywords- Power quality disturbances; HHT; S-transform; Signal classification

\section{INTRODUCTION}

Along with the wide application of power electronic equipments, the power quality of distribution grid is polluted by various types of non-linear and impact load. At the same time, supplying higher power quality is also required by significantly increased detection, analysis and control of electrical equipments, which is based on computer and microprocessor. Power quality detection is the most important prerequisite for improving power quality ${ }^{[1]}$.In order to classify the disturbance signals correctly, signal feature extraction is needed. The usual approach is primarily based on the transformation and reconstruction of the original waveform, extracting classification features, impelling a large number of signal processing methods applied to the detection of power quality problems. The most commonly used feature extraction method is Wavelet Transform ${ }^{[2-7]}$. Fourier Transform ${ }^{[4]}$, dq Transform ${ }^{[8]}$, Stransform ${ }^{[9-12]}$, Walsh Transform ${ }^{[11]}$ and $\mathrm{HHT}^{[13]}$ were also used for disturbance features extraction.

Currently, most studies of the power quality problems is for single disturbance, however, studies involve the analysis of composite disturbances is rare. The literature [14] [15] [16 discussed the harmonics and sag, harmonics and swell. In addition, the literature [17] is also referred to a composite disturbance during the data analysis process, but did not indicate the specific type. In literature [18], based on mathematical morphology and grille fractal, a novel approach on power quality disturbance detection and location is presented. S-transformation is a time-frequency analysis method developed from CWT combining with STFT. It brings in the Gaussian window in which the width and frequency changes reversely and has the relevant resolution with frequency. HHT absorbed the advantages of Wavelet Transform's multi-resolution and overcame difficulties in selecting wavelet basis ${ }^{[14]}$, this method accessed the concept of the base function from the signal itself, which has a good self-adaptability ${ }^{[15]}$ directly.

In this paper, Matlab is used to simulate six kinds of single power quality disturbance signal and eight kinds of composite disturbance signal, including voltage sags, voltage swells, voltage interruption, transient shocks, voltage flicker, voltage harmonics, and composite disturbances, witch are created by mixing long-term disturbances(harmonics, flicker) and short-term disturbances (voltage swells, voltage sags, voltage interruption, transient shocks), in order to acquire the corresponding disturbance signals. We can get the instantaneous amplitude of these signals before and after the disturbance moment by means of HHT. On the other hand, we can use the result of S-transform of the disturbance signals to get the high-frequency and low-frequency characteristics to accurately detect power quality disturbance signals' categories and disturbance characteristics, as well as disturbance signals' starting and ending time.

\section{HHT}

HHT is a non-linear analysis method, which can be used for transient signals. The method is composed of EMD and Hilbert transform, the core part is EMD.

\section{A. EMD decomposition and IMF function}

EMD method assumes that every signal is formed by different intrinsic mode functions (IMFs) and each IMF is a set sequence of smooth linear or nonlinear data. Signal is decomposed into a set of intrinsic mode functions by EMD: 


$$
x(t)=\sum_{i=1}^{n} i m f_{i}(t)+r_{n}(t)
$$

\section{B. Hilbert transform}

The most important feature of HHT method is that each IMF component can be Hilbert transformed directly, in order to achieve the extraction of the signal's instantaneous parameters. Hilbert transform is defined as follows:

$\mathrm{X}(\mathrm{t})$ is a continuous time series:

$$
\mathrm{Y}(\mathrm{t})=\frac{1}{\pi} \int_{-\infty}^{+\infty} \frac{\mathrm{X}(\tau)}{\mathrm{t}-\tau} \mathrm{d} \tau=\frac{1}{\pi} \int_{-\infty}^{+\infty} \frac{\mathrm{X}(\mathrm{t}-\tau)}{\tau} \mathrm{d} \tau=\mathrm{x}(\mathrm{t}) * \frac{1}{\pi \mathrm{t}}
$$

$Y(t)$ can be regarded as the output of the filter of $x(t)$, and the inverse transform is:

$$
X(t)=\frac{1}{\pi} \int_{-\infty}^{+\infty} \frac{Y(\tau)}{\tau-t} d \tau
$$

Analytical form:

$$
\mathrm{Z}(\mathrm{t})=\mathrm{X}(\mathrm{t})+\mathrm{j} Y(\mathrm{t})=\mathrm{a}(\mathrm{t}) \mathrm{e}^{\mathrm{j} \theta(\mathrm{t})}
$$

$a(t)$ is the instantaneous amplitude,

nstantaneous phase. The instantaneous frequency is:

$$
f(t)=1 / 2 \pi(d \theta(t)) / d t
$$

\section{THE BASIC PRINCIPLES OF S-TRANSFORM}

S-transform was put forward by U.S. geophysicist Stockwell in 1996[12], concordance the advantages of shorttime Fourier transform and Wavelet transform and avoided their shortcomings. It is a linear, multi-resolution and nondestructive reversible time-frequency analysis method.

$\mathrm{S}$-transform (ST) of the signal $\mathrm{h}(\mathrm{t})$ is defined as:

$$
\mathrm{S}(\tau, f)=\int_{-\infty}^{+\infty} h(t) \frac{|f|}{\sqrt{2 \pi}} \exp \left[-\frac{1}{2}(\tau-t)^{2} f^{2}\right] \exp (-i 2 \pi f t) d t
$$

$\frac{|f|}{\sqrt{2 \pi}} \exp \left[-\frac{1}{2}(\tau-t)^{2} f^{2}\right]$ is Gaussian window; $\tau$ controls the location of Gaussian window on the time axis $t$; $f$ is for the frequency; $\mathrm{i}$ is the imaginary unit. We can see that $\mathrm{S}$ transform is different from the short-time Fourier transform, as the height and width of the Gaussian window varies with frequency. It overcomes the defect that the height and width of STFT window is fixed.

Formula (6) can be discreted:

$$
\begin{gathered}
\mathrm{S}(m, n)=\sum_{k=0}^{N-1} H(n+k) \exp \left(-\frac{2 \pi^{2} k^{2}}{n^{2}}\right) \exp (i 2 \pi k m / N), n \neq 0 \\
\mathrm{~S}(m, n)=\frac{1}{N} \sum_{k=0}^{N-1} h[k], n=0 \\
H(n)=\frac{1}{N} \sum_{k=0}^{N-1} h[k] \exp (-i 2 \pi k n / N)
\end{gathered}
$$

For the discrete signal of $\mathrm{N}$ points $\mathrm{h}[\mathrm{i}](\mathrm{i}=0,1,2, \ldots, \mathrm{N}$ 1), we can get the $S$-transform result as a matrix of $n+1$ rows and $\mathrm{m}$ columns using the formula(7) and (8),referred to as the $\mathrm{S}$ matrix. The columns of $\mathrm{S}$ matrix correspond to the sampling time points, the lines correspond to the sampling frequencies. The first row $(n=0)$ corresponds to the $D C$ component of the signal and the frequency difference between adjacent rows is:

$$
\Delta f=\frac{f_{s}}{N}, f_{n}=\frac{f_{s}}{N} n
$$

$\mathrm{N}$ is the number of samples, fs is the sampling frequency.

$\mathrm{S}$ modular matrix is obtained by module operation for each element of the $\mathrm{S}$ matrix. Its column vector represents the signal's amplitude-frequency characteristic at a particular moment. the row vector represents the time domain distribution of signal in a certain frequency component.

\section{FEATURE EXTRACTION OF POWER QUALITY DISTURBANCES}

In this paper, the fourteen kinds of power quality disturbance characteristics are conformed to the IEEE standard $^{[16]}$ and evenly distributed, including voltage sags, voltage swells, voltage interruption, transient shocks, voltage flicker, voltage harmonics, and composite disturbances, witch are created by mixing long-term disturbances (harmonics, flicker) and short-term disturbances(voltage swells, voltage sags, voltage interruption, transient shocks). Various power quality disturbance signals are randomly generated, which is similar to the literature [22].
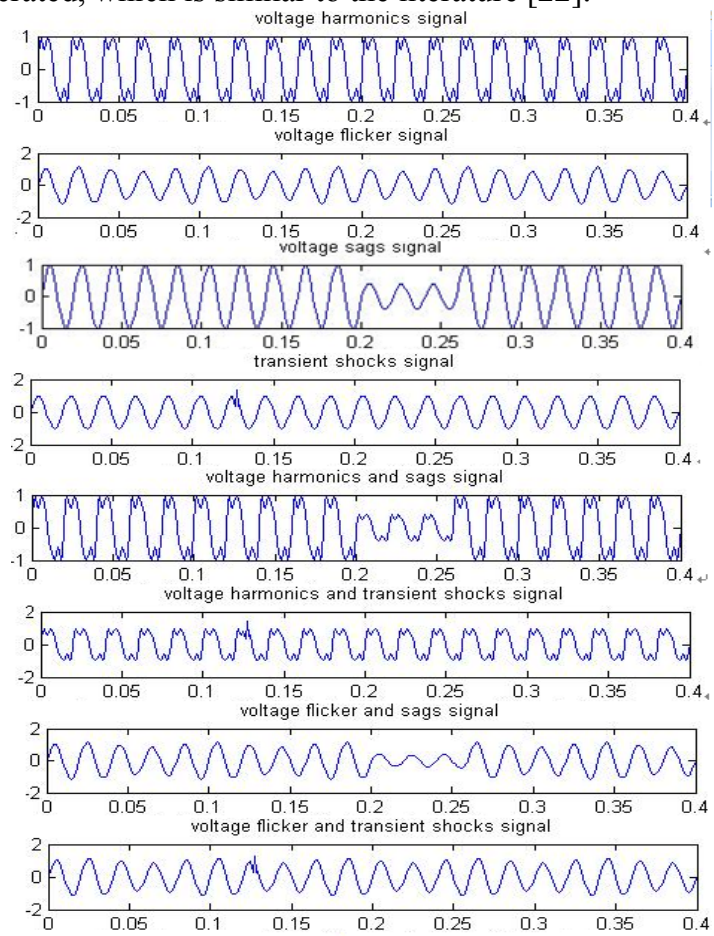

Figure 1. Figure1. Power quality disturbance signals

They are generated by Matlab, the sampling frequency is $1 \mathrm{kHz}$, the voltage fundamental frequency is $50 \mathrm{~Hz}$ and the simulation length is $0.4 \mathrm{~s}$. Among them, the voltage amplitude of voltage sags is randomly generated from 0.1 (p.u.) to 0.9 (p.u.), the voltage amplitude of voltage swells is randomly generated from 1.1(p.u.) to 1.8(p.u.). The 
starting and ending times of short-term disturbances are randomly generated within a predetermined range, as shown in Figure 1. The analysis principles and performance of image for voltage sags have a similar nature with voltage swells and voltage interruption, so in Figure 1, Figure 2, Figure 3 and Figure 4, we just take voltage sags as a representative.

The $\mathrm{S}$ modular matrix can express the changes from low frequency to high frequency of signals' characteristic clearly and intuitively, so it is available to use the S-transform for the feature extraction of power quality disturbance signals. After the transformation of the signal by HHT, it can clearly reflect the instantaneous amplitude of the voltage signals before and after the disturbance moment. The disturbance's starting and ending time of short-term signals can be worked out by the instantaneous frequency of imfl, which is obtained by the result of EMD. We can also get them by the high-frequency amplitude characters of $\mathrm{S}$ modular matrix. For the convenience of features' extraction, the following characteristic functions and values are defined:

- The function of amplitude of the time-varying fundamental frequency $V_{f b}(t)$

$V_{f b}(t)$ is defined in $\mathrm{S}$ modular matrix, corresponding to the amplitude of the fundamental frequency $(50 \mathrm{~Hz}$ in this article) changing over time.

$$
V_{f b}(t)=S_{a}\left(t, f_{b}\right)
$$

Where $t$ represents the sampling time, $f b$ is the fundamental frequency $(50 \mathrm{~Hz}$ in this article).

- The average value of amplitude for the fundamental frequency $F_{\text {bav }}$

$$
F_{\mathrm{bav}}=\frac{1}{L} \sum_{l=1}^{L} S_{a}\left(t, f_{b}\right)
$$

$\mathrm{L}$ is the number of samples.

- Maximum/minimum of fundamental frequency's amplitude $F_{\text {bmax }} / F_{\text {bmin }}$

$$
F_{\mathrm{bmax}}=\max \left[V_{f b}(t)\right] \quad F_{\mathrm{b} \text { min }}=\min \left[V_{f b}(t)\right]
$$

- Characteristic time-varying function of highfrequency's amplitude $V_{f h}(t)$

$V_{f h}(t)$ is defined in $\mathrm{S}$ modular matrix, corresponding to the mean square of the amplitude of the high frequencies changing over time. The minimum frequency value is more than two times the fundamental frequency's, and its expression is as follows:

$$
V_{f h}(t)=\frac{1}{n-m} \sum_{f_{h}=m}^{n}\left[S_{a}\left(t, f_{h}\right)^{2}\right]
$$

For $\mathrm{Sa}, \mathrm{n}$ is the total number of rows, that is, the number of frequencies which can be analyzed; $m$ is the row's number of the frequency whose value is 2 times of the fundamental frequency's. The mold square for frequency values in the matrix is to reduce the impact of noise in the signal processing.

- Mean of high-frequencies' amplitude in S modular matrix
It is defined as the mean of $\mathrm{Vfh}(\mathrm{t})$, namely, it is the mean square of the high-frequency component's amplitude in the entire time period, as the following expression shows:

$$
F_{\text {hav }}=\frac{1}{L} \sum_{l=1}^{L} V_{f h}(t)=\frac{1}{L} \sum_{l=1}^{L} \frac{1}{n-m} \sum_{f_{h}=m}^{n}\left[S_{a}\left(t, f_{h}\right)^{2}\right]
$$

- Maximum of Characteristic time-varying function of high- frequency's amplitude $F_{h \max }$

$$
F_{\text {hmax }}=\max \left[V_{f h}(t)\right]
$$

- The short-term disturbance's starting time Ts, ending time $\mathrm{Te}$

The short-term disturbance's starting time is Ts and the termination time is $\mathrm{Te}$

- The instantaneous amplitude's flatness during nontransient disturbance moment Fba:

$$
F_{b a}=\left|\left(A F_{\text {max }}-1\right)-\left(A F_{\text {min }}-1\right)\right|
$$

Where $A F_{\text {min }} / A F_{\text {max }}$ is the maximum/ minimum of the instantaneous amplitude during non-transient disturbance moment.

- Degree of change of the fundamental frequency's amplitude Fmm

If the signal is a stable sine wave, its amplitude of the fundamental frequency would always be 1 by S-transform . The characteristic function is defined as the maximum amplitude of the fundamental frequency minus the minimum one:
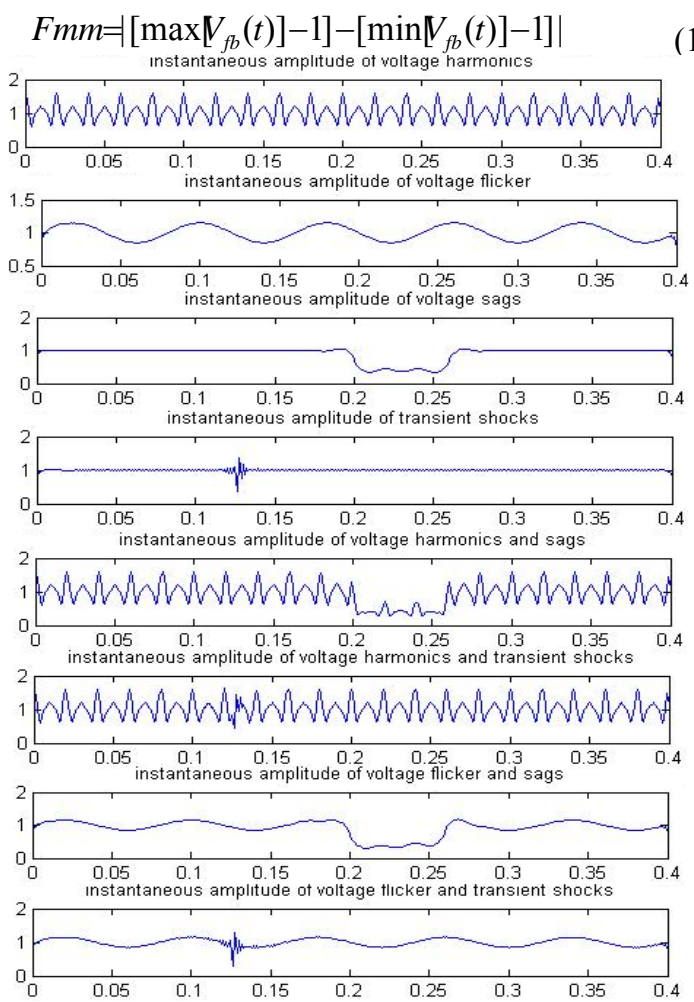

Figure 2. Instantaneous amplitude of disturbance signals after HHT 
Figure 2 shows the instantaneous amplitude of power quality disturbance signals after HHT. From the diagram, we can see the corresponding changing process of signal's amplitude. After S-transform, Figure 3 and 4, respectively
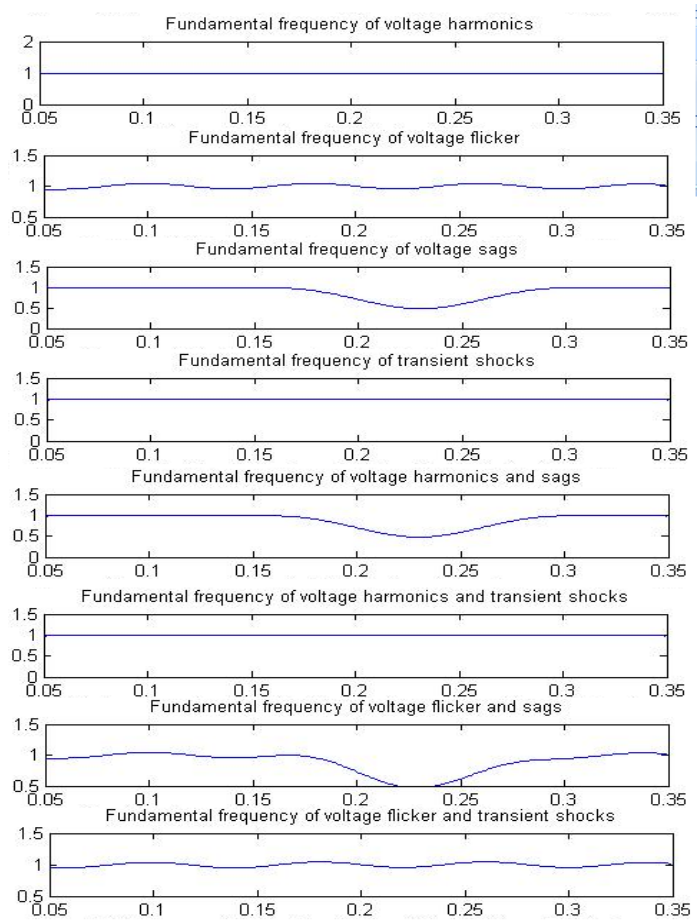

Figure 3. Fundamental frequency of disturbance signals after S-transform
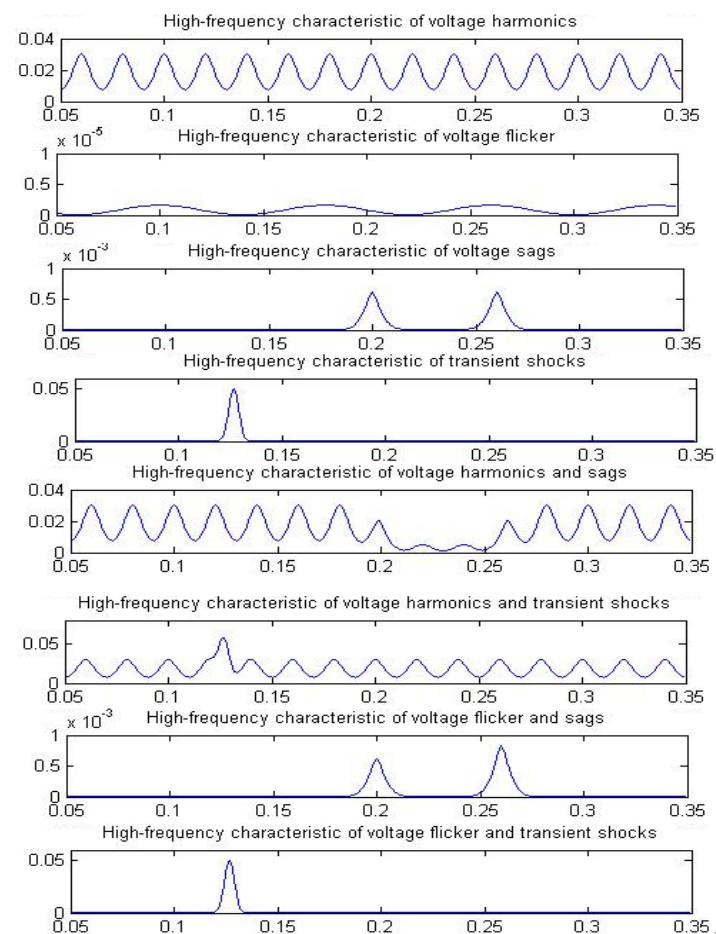

Figure 4. High-frequency characteristic of disturbance signals after Stransform show the fundamental frequency amplitude curve and the high-frequency time-varying function $\mathrm{Vfh}(\mathrm{t})$ curve of the disturbance signals. From the two plots, we can see the fundamental frequency's characteristic, the size of the highfrequency component and the corresponding time, which are contained in disturbance signals. In Figures 2, 3 and 4, we have omitted the end data portions of the image, in order to avoid the impact of end effect, so did the algorithm in this article.

Finally, complete content and organizational editing before formatting. Please take note of the following items when proofreading spelling and grammar:

\section{RECOGNITION AND CLASSIFICATION OF POWER QUALITY DISTURBANCE SIGNALS}

The disturbance signals' amplitude, containing voltage sags and voltage interruption, shows a cave shape, the instantaneous amplitude of signals, containing voltage swells, shows a convex shape. According to the standard for the description of the various power quality disturbances[23], which is recommended by IEEE 22 Standard Commission, classification as follows (refer to Figure 5): The value of disturbance signal's Fbav, including voltage sags or interruption, will be significantly lower than the corresponding values of the other, in this article, we take 0.99 as the demarcation point to complete the classification 1-1; According to the power system electromagnetic phenomena characteristic parameters and classification, developed by the IEEE, the frequency components of voltage harmonic present in the entire time period, while the highfrequency components of the transient shocks exist only within a short time (less than $50 \mathrm{~ms}$ ). Therefore, the mean of high-frequencies' amplitude Fhav of voltage harmonics is much larger than the other corresponding values, 0.005 is taken as the cut-off point to complete classification 2-2, 3-1 and 3-2; The decline values of disturb signals containing voltage interruption have varying degrees with sags, which can be distinguished by Fbmin, we take the absolute value 0.1 as the cut-off value to complete classification 3-3 and 3-4; The Fba of disturber signals containing voltage flicker, the instantaneous amplitude's flatness during non-transient disturbance moment, is significantly higher than other signals', 0.05 is the cut-off value in this article , completing classification 4-2,4-3,5-2; The Fbmax of signals containing voltage flicker or swells, maximum of fundamental frequency's amplitude, will be higher than the other signals', this paper take 1.01 as categorical values to complete the classification 2-1; In the classification 4-1, Fmm of signals containing voltage swells will be significantly higher than the others', the value of 0.1 could be the point to achieve this classification; Voltage signals containing transient oscillation will have a large number of high-frequency components in a short time, however, voltage containing flicker does not. We take 0.001 of Fhmax as the demarcation point for classification 5-1.

According to the above classification process, the logic for classification and identification of the fourteen power quality disturbance signals is given, as shown in Figure 5: 


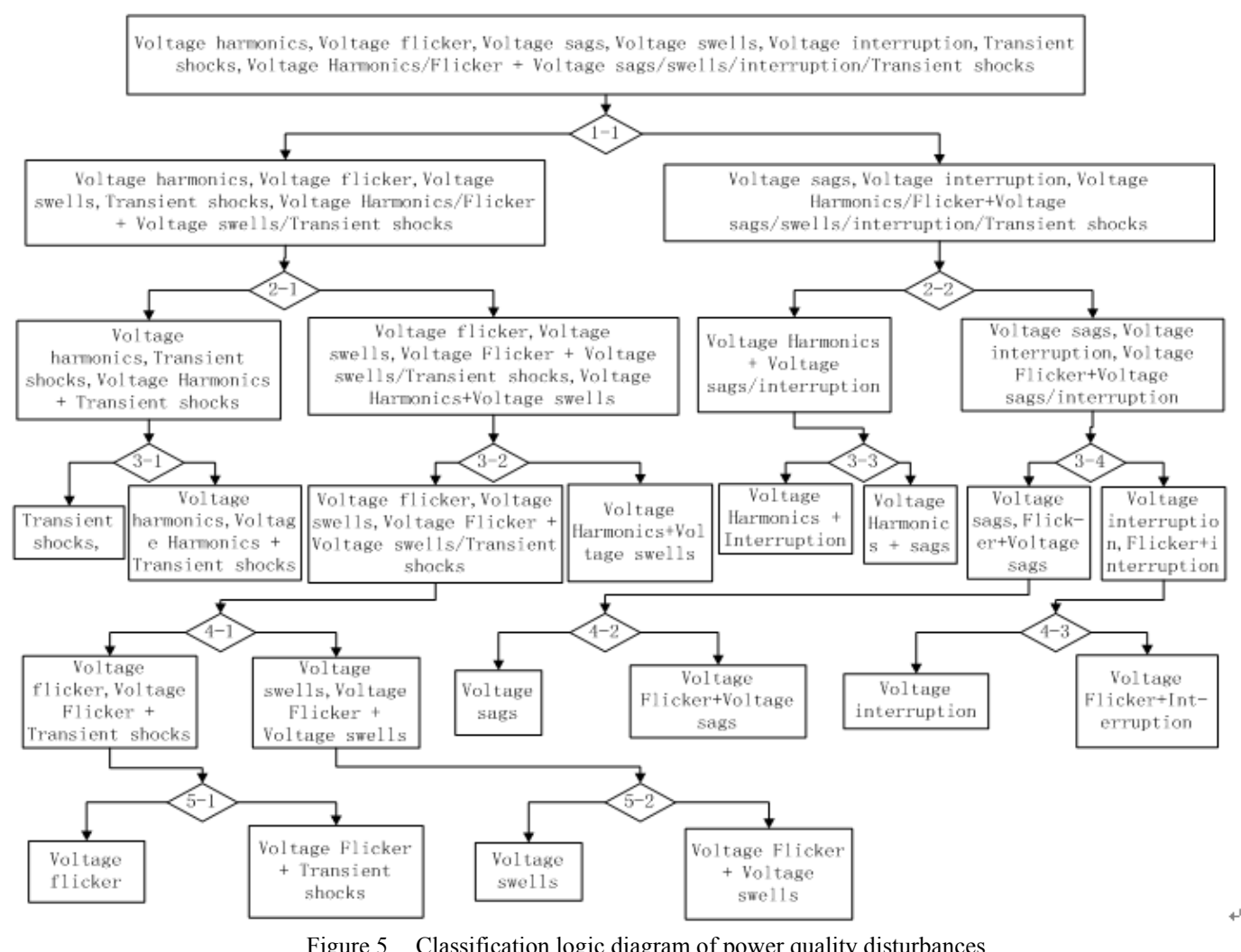

In order to verify the effectiveness of the method of this classification, the article conducts several experiments in which the signals are randomly generated within a

predetermined range. Table 1 illustrates one set of experimental data.

TABLE I. EXPERIMENTAL DATA

\begin{tabular}{|c|c|c|c|c|c|c|c|c|c|}
\hline Eigenvalues & Fbav & Fbmax & Fhav & Fbmin & Fmm & Fba & Fhmax & Ts & $\mathrm{Te}$ \\
\hline Voltage harmonics & 0.9986 & 0.9999 & 0.0178 & 0.9756 & 0.0243 & 0.9711 & 0.0304 & $1 e-4$ & 0.400 \\
\hline Voltage flickers & 0.9985 & 1.0433 & $8.319 \mathrm{e}-7$ & 0.9461 & 0.0972 & 0.3011 & $1.6145 \mathrm{e}-6$ & 1e-4 & 0.400 \\
\hline Voltage sags & 0.8795 & 0.9999 & $4.0828 \mathrm{e}-5$ & 0.4789 & 0.5210 & 0.0073 & $6.1644 \mathrm{e}-4$ & 0.194 & 0.270 \\
\hline Voltage swells & 1.1183 & 1.5209 & $4.1104 \mathrm{e}-5$ & 0.9756 & 0.5453 & 0.0107 & $6.1714 \mathrm{e}-4$ & 0.155 & 0.229 \\
\hline Voltage interruption & 0.7396 & 0.9997 & $9.1632 \mathrm{e}-4$ & 0.0703 & 0.9294 & 0.0102 & 0.0181 & 0.157 & 0.254 \\
\hline Transient shock & 0.9966 & 0.9999 & $7.7766 \mathrm{e}-4$ & 0.9755 & 0.0243 & 0.0322 & 0.0504 & 0.112 & 0.145 \\
\hline Harmonics and sags & 0.8796 & 0.9999 & 0.0149 & 0.4791 & 0.5207 & 0.9690 & 0.0304 & 0.190 & 0.268 \\
\hline Harmonics and swells & 1.1183 & 1.5206 & 0.0234 & 0.9756 & 0.5450 & 0.9746 & 0.0779 & 0.151 & 0.226 \\
\hline $\begin{array}{l}\text { Harmonics and } \\
\text { interruption }\end{array}$ & 0.7405 & 0.9997 & 0.0137 & 0.0704 & 0.9294 & 0.9694 & 0.0366 & 0.163 & 0.261 \\
\hline $\begin{array}{l}\text { Harmonics and } \\
\text { transient shock }\end{array}$ & 0.9966 & 0.9999 & 0.0188 & 0.9756 & 0.0243 & 0.9967 & 0.0580 & 0.110 & 0.147 \\
\hline Flicker and sags & 0.8832 & 1.0433 & $4.8272 \mathrm{e}-5$ & 0.4731 & 0.5702 & 0.3008 & $8.2559 \mathrm{e}-4$ & 0.196 & 0.268 \\
\hline Flicker and swells & 1.1216 & 1.5856 & $3.5885 \mathrm{e}-5$ & 0.9461 & 0.6394 & 0.3034 & $6.3390 \mathrm{e}-4$ & 0.157 & 0.223 \\
\hline Flicker and interruption & 0.7403 & 1.0423 & $9.905 \mathrm{e}-4$ & 0.0710 & 0.9713 & 0.2981 & 0.0196 & 0.158 & 0.251 \\
\hline $\begin{array}{c}\text { Flicker and } \\
\text { transient shock }\end{array}$ & 0.9965 & 1.0433 & $9.765 \mathrm{e}-4$ & 0.9461 & 0.0972 & 0.3145 & 0.0504 & 0.115 & 0.139 \\
\hline
\end{tabular}

It can be concluded that the above experimental data of power quality disturbances can accurately classify the types of disturbance signals according to the above logic method. We can also observe the amplitude before and after the disturbance moment, starting and ending time based on the detection data in the table.

\section{SUBMISSION}

This paper extracts the instantaneous amplitude as well as the low-frequency and high-frequency characteristics of the disturbance signals, basing on the HHT and S-transform of thirteen kinds of power quality disturbance signals including 
six kinds of single power quality disturbance signals and eight kinds of composite disturbance signals. We also have accurately detected the categories and characteristics of the disturbance signals, as well as their starting and ending time. Multigroup experimental results have showed that the classification logic in this paper is appropriate for the thirteen kinds of power quality disturbance signals' classification, meanwhile, we need to further study that how to distinguish voltage harmonics and voltage harmonics with transient shocks.

\section{ACKNOWLEDGEMENTS}

This work is supported by Key scientific and technological project of China Southern Power Grid Company (K-GD2012-343)

\section{REFERENCES}

[1] Mc Graw Hill. Electrical power systems quality. New York, 2002:1 52.

[2] YANG Jin, LI Chengyin, ZHOU Ming. The application of generalized interpolating wavelet analysis in the power quality disturbance signals. Electric Power Automation Equipment, 27(1):2529,2007 .

[3] FANG Guozhi, HU Bingfang, SONG Guoyi.The detection method of three typical grid disturbance signals. Electric Power Automation Equipment, 28(6):74-76,2008.

[4] Liao Y, Lee J B. A fuzzy-expert system for classifying power quality disturbances. International Journal of Electrical Power and Energy Systems, 26(3) : 199-205, 2004.

[5] MEI Xue, WU Weilin. Power quality classification based on wavelet and ANN. Journal of Zhejiang University: Engineering Science , 38(10):1383-1386,2004.

[6] HE H B, Starzy J A. A self-organization learning array system for power quality classification based on wavelet transform. IEEE Transactions on Power Delivery, 21(1): 286-295, 2006.

[7] SHI Min,WU Guozheng, XU Xi. Automatic identification for power quality disturbances based on probabilistic neural networks and double wavelet. Electric Power Automation Equipment, 26(3):5-8, 2006.

[8] ZHANG Xiujuan, XU Yonghai. The power quality disturbance detection and identification method based on $\mathrm{dq}$ transform and wavelet transform. Electric Power Automation Equipment, 25(7):1-5, 2005.

[9] Chiluku R I M V, Dask P K. Multi-resolution S-transform based fuzzy recognition system for power quality events. IEEE Transactions on Power Delivery, 19(1): 323-330, 2004.
[10] Lee I W C, Dash P K. S-transform based intelligent system for classification of power quality disturbance signals. IEEE Transaction on Power Delivery, 18(3): 800-805, 2003.

[11] SONG Xiaofang, CHEN Jincao. Classification method for dynamic power quality disturbances based on supported vector machine. Electric Power Automation Equipment, 26(4): 39-42, 2006.

[12] Stockwell R G, Mansinha L, Lowe R P. Localization of the complex spectrum: the S-transform [J]. IEEE Transactions on Signal Processing, 44(4): 998-1001, 1996.

[13] LI Wenfan,LIU Zhigang,SUN Wanlu. The system of power quality monitoring based on HHT. Power System Protection and Control, 39( 23): 123-127, 2011

[14] Gaouda A M, Kanoun S H, Salama M M A, etal. Pattern Recognition Applications for Power System Disturbance Classification [J].IEEE Transaction on Power Delivery, 2002(3):677 - 683.

[15] Mishra S, Bhende C N, Panigrahi B K. Detection and classification of Power Quality Disturbances Using S-Transform and Probabilistic Neural Network[J].IEEE Transaction on Power Delivery, 2008(1):280-287.

[16] He H, Starzyk J A. A self-organizing learning array system for power quality classification based on wavelet transform[J].IEEE Transaction on Power Delivery, 2006(1):286-295.

[17] Monedero I, Leon C, Ropero J, et al. Classifieation of Electrical Disturbances in Real Time Using Neural Networks[J].IEEE Transactions on Power Delivery, 2007(3): 1288-1296.

[18] LI Gengyin, LUO Yan, ZHOU Ming. The detection and localization of power quality disturbances based on mathematical morphology and grille fractal [J]. China Electrical Engineering, 2006(3):25-30.

[19] YANG Cunxiang, TONG Zhanying, WAN Yuhao. The extraction and research of power system transient composite disturbance signals based on HHT [J]. Power System Protection and Control, 2009, 37 (11) : 6-9.

[20] WANG Bo, YANG Honggeng. A new detection method for shortterm disturbances in power system based on EMD and TEO [J]. Relay, 2006, 34 (8): 56 - 61.

[21] Standards Coordinating Committee 22 on Power Quality. IEEE Recommended Practice for Evaluating Electric Power System Compatibility With Electronics Process Equipment [S], 1998, IEEE Std, 1346-1998.

[22] Abdel- Galil T K, Kamel M. Power quality disturbance classification using the inductive inference approach [J]. IEEE Trans- actions on Power Delivery, 2004, 19(4): 1812-1818.

[23] IEEE. Standards Coordinating Committee 22 on Power Quality. IEEE Recommended Practice for Monitoring Electric Power Quality. 1995. 